\title{
Space Charge Modelling in HVDC Extruded Cable Insulation
}

\author{
Yunpeng Zhan, George Chen, Miao Hao \\ University of Southampton \\ Highfield, Southampton \\ SO17 1BJ, UK
}

\begin{abstract}
With the growing interest in the development of HVDC cable technologies, the impact of space charge accumulation inside the polymeric insulation has attracted more attentions and the field distribution across the insulation has become the focus point of research. In this paper, several modifications have been applied on the bipolar charge transport model developed for the cable geometry. Based on many experimental observations, a threshold electric field at which the charge injection takes place was introduced. Considering the practical operation of HVDC cables, the thermal transient effects on space charge behaviors were particularly taken into account. A changing current flow has been applied in the core of a MV size cable, in order to further study the charge dynamics and to anticipate the field distribution within the insulation. The simulation results suggest that the thermal transient can affect the amount of injected charge and the charge movement significantly. The field inversion can only take place with higher temperature and larger temperature gradient, and this can be maintained even with temperature decreasing.
\end{abstract}

Index Terms - HVDC cable, polymeric insulation, bipolar charge transport model, temperature gradient, thermal transient, field inversion.

\section{INTRODUCTION}

INCREASING reliance on renewable sources of electricity generation accelerates the development of high voltage direct current (HVDC) transmission technologies [1]. Compared with overhead lines, HVDC cable technologies are more recommended such as offshore windfarm and sea crossing. Many different HVDC cable technologies have been developed in preceding decades and have been applied successfully around the world. Although the majority of well-established HVDC cable systems currently in service are based on paper/oil or mass impregnated insulation systems, HVDC cables using extruded polymeric insulation have attracted more interests due to its environmental benefits, economical production and higher operating temperature [2]. Most developments in HVDC cables have been focused on the electric field distribution within the main insulation. Unlike $\mathrm{AC}$ cables, the electric field distribution is more complex under DC condition due to not only the field and temperature dependent conductivity, but also the space charge effects. The accumulated space charge can distort the electric field distribution in the insulation, leading to accelerated aging and eventual breakdown $[3,4]$. Issues related to space charge behavior need to be further studied, in order to enhance the reliability and lifetime of HVDC extruded cables.

Many numerical models have been proposed to investigate the polymeric material behavior in DC insulating systems. These

Manuscript received on X Month 2005, in final form XX Month 2005. models can be generally classified into two types, the one which simulates the macroscopic material behavior based on conductivity data obtained as a function of temperature and electric field, the other one which describes the microscopic space charge dynamic characteristics inside the dielectrics [5-7]. The simulation results from the both kinds of models, when compared with the experimental results, can reasonably represent charge density profiles and electric field distributions in the bulk of dielectrics and their evolving with time. However, compared with models that based on the varying conductivity, charge transport model is more physical as it describes charge carriers transport process in the dielectrics (including charge trapping, detrapping and recombination), and especially considers the charge injection at the interface between the electrodes and the dielectric. Many researchers have further developed this kind of models in order to achieve a better fit with experimental data, and most attempts have been made to describe the charge dynamics in the planar samples [8]. So far, few researchers take into account the cable geometry factor in their models, which has a great impact on the electric field distribution in the insulation due to the divergent field. Additionally, the temperature gradient across the insulation also needs to be considered for its impact on charge generation and transportation. In 2016, Le Roy et al. extended the bipolar charge transport model to a cylindrical configuration, considering a steady temperature gradient across the insulation [9]. The simulated results show how the geometry and the temperature can respectively affect the charge densities and electric field distributions. However, in practical 
HVDC application, the temperature distribution across the insulation can be affected by the current flowing in the conductor. The current flowing through the conductor varies with the transmission power and this will lead to the change in temperature gradient across the insulation. Therefore, a more complex model needs to be developed when considering the thermal transient effects caused by the flowing current in the conductor.

In this paper, a modified bipolar charge transport model has been proposed to simulate space charge behaviour in cable geometry insulation. Particularly, the effects of thermal transient on the space charge dynamic has been considered by applying a current flow in the core of cable. The evolution of space charge and electric field have been studied with the varying temperature distribution and field. The comparisons will be made between the previous model and this updated one. Moreover, the space charge behaviour with a changing current flowing in the core of cable has been studied, in order to investigate if the model can be used to describe the specific characteristics in the practical operation.

\section{BRIEF SUMMARY OF THE MODEL}

Due to the significant improvement of fabrication and purification on polymeric insulation, space charge generated by ionization process are much less than those from electronic injection under high electric field [10,11], therefore in the numerical modelling, charge generation mainly depends on charge injection and the dissociation is neglected. The bipolar charge transport model, which has been presented elsewhere [8], starts from the injection of positive and negative charge carriers at the interface between the insulation and the electrodes when the applied electric field exceeds the threshold field. The injected electrons and holes move into the bulk of dielectrics towards the opposite electrodes under the influence of the electric field hence come the conduction current. The trap energy levels are localized in the band-gap of the dielectrics. Shallow traps originated by physical defects and deep traps caused by chemical defects in the molecules can capture the mobile carriers and form trapped carriers inside [12]. Therefore, there are four species considered in the model, mobile electrons and holes, trapped electrons and holes, as shown in Figure 1.

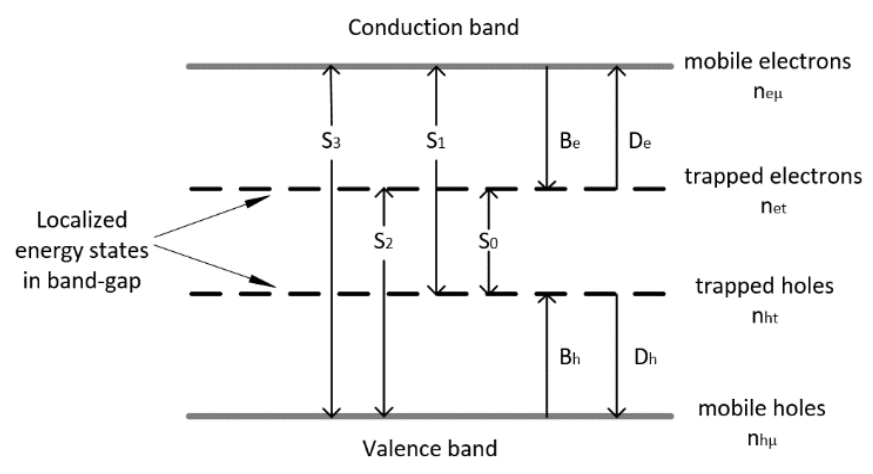

Figure 1. Schematic representation of the bipolar charge transport model. $\mathrm{S}_{\mathrm{i}}$, $B_{i}$ and $D_{i}$ are recombination, trapping and de-trapping coefficients respectively. $\mathrm{n}_{\mathrm{i}}$ is the charge density. Indexes e and $\mathrm{h}$ refer to electrons and holes; $\mu$ ant $t$ refer to mobile and trapped charge carriers [6].

Figure 2 (a) shows the cable geometry and Figure 2 (b) presents its schematic discretization grids used for the simulation. For the numerical computation, the thickness of the sample is divided into 200 divisions of different sizes of thickness $\Delta \mathrm{x}$ (the distance between arcs in Figure 2 (b)). In order to optimize the simulation results, the $\Delta \mathrm{x}$ is set to be smaller close to the electrodes. The smallest cell $\Delta \mathrm{x}$ next to the electrodes is of the order of $550 \mathrm{~nm}$, and the maximal is about 100 times as thick as the smallest one.

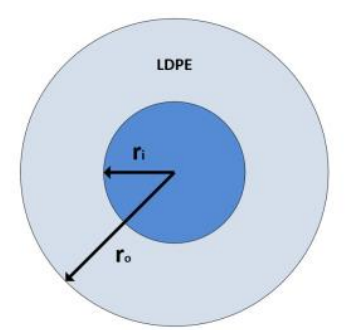

(a)

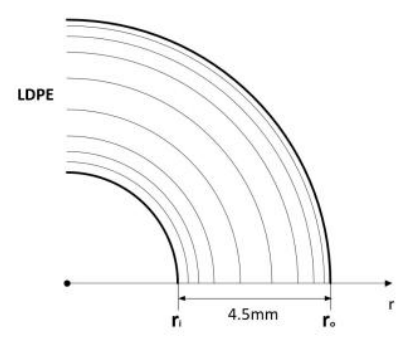

(b)
Figure 2. The schematic representation of the cable geometry (a) and the 1dimensional grids used for the simulations (b).

To describe dynamics of charge accumulation in the polyethylene insulation, three essential space and time dependent equations are presented in cylindrical geometry as following, neglecting diffusion,

\section{Poisson's equation (in cylindrical coordinates):}

$$
\frac{\partial^{2} V(r, t)}{\partial r^{2}}+\frac{1}{r} \frac{\partial V(r, t)}{\partial r}=-\frac{\rho(r, t)}{\varepsilon_{0} \varepsilon_{r}}
$$

\section{Continuity equation:}

$$
\frac{\partial n_{a \mu, a t}(r, t)}{\partial t}+\frac{1}{r} \frac{\partial\left(j_{a} \cdot r\right)}{\partial r}=s_{a \mu, a t}(r, t)
$$

Ohmic's Law:

$$
j_{a}(r, t)=\mu_{a}(r, t) n_{a \mu}(r, t) E(r, t)
$$

where $j_{a}$ is the conduction current density; $n$ is the charge density; $E$ is the electric field; $\rho$ is the net charge density; $\varepsilon_{O}$ is the vacuum permittivity, $\varepsilon_{r}$ is the relative dielectric permittivity, which is considered as a constant ( 2.3 for polyethylene). Here $a$ refers to the type of charge, and the subscript $\mu$ or $t$ refers respectively to mobile or trapped charge. $s$ are the source terms, which encompass the changes in local density by the processes other than transport (trapping, detrapping and recombination).

The conduction between shallow traps is described using a hopping type mobility as a function of field and temperature [9]. The impacts of electric field and temperature on the mobility for holes and electrons are shown in Figure 3 (a) and (b) separately. It can be clearly observed that the variation of temperature from $25^{\circ} \mathrm{C}$ to $65^{\circ} \mathrm{C}$ has a larger impact on the value of the mobility (increase by more than one order of magnitude) compared with the variation of electric field from $10 \mathrm{kV} / \mathrm{mm}$ to $40 \mathrm{kV} / \mathrm{mm}$. Additionally, the detrapping coefficients and the recombination coefficients are also functions of temperature and they have been described already in literature [9].

The injection of charge carriers occurs at the interface between the conductor and the insulator when the applied electric field is higher than the threshold field. From the previous work done 
by Liu et al. [13], a value of threshold electric field about 10 $\mathrm{kV} / \mathrm{mm}$ has been identified for charge injection in polyethylene. In this study, when the applied field exceeds the critical value $(10 \mathrm{kV} / \mathrm{mm})$, the boundary condition is defined by the Schottky injection at both electrodes.

$$
\begin{aligned}
& j_{e}\left(r_{o}, t\right)=A T^{2}(r) \exp \left(\frac{-e w_{e i}}{k_{B} T(r)}\right) \exp \left(\frac{e}{k_{B} T(r)} \sqrt{\frac{e E(r, t)}{4 \pi \varepsilon_{0} \varepsilon_{r}}}\right) \\
& j_{h}\left(r_{i}, t\right)=A T^{2}(r) \exp \left(\frac{-e w_{h i}}{k_{B} T(r)}\right) \exp \left(\frac{e}{k_{B} T(r)} \sqrt{\frac{e E(r, t)}{4 \pi \varepsilon_{0} \varepsilon_{r}}}\right)
\end{aligned}
$$

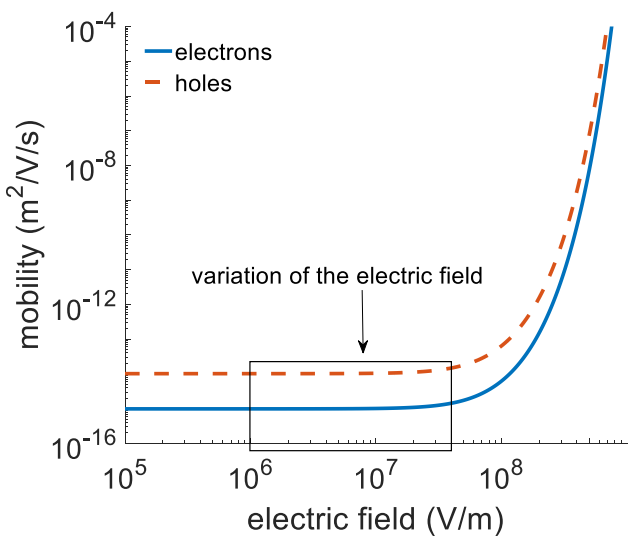

(a) Simulated mobility as a function of electric field at $25^{\circ} \mathrm{C}$

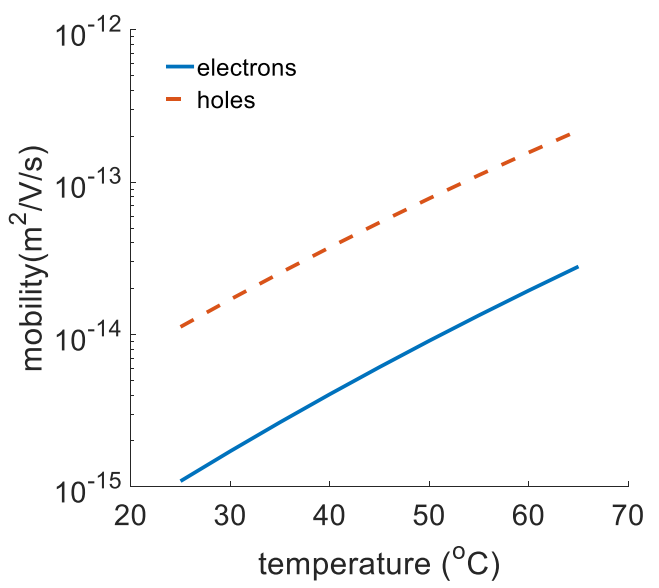

(b) Simulated mobility as a function of temperature for $\mathrm{E}=20 \mathrm{kV} / \mathrm{mm}$

Figure 3. Simulated mobility as a function of field and temperature. Values of trap depths for the mobility can be found in Table 1 [9].

However, there are still few injected space charges can be detected when the bulk is subjected to relatively low electric field. Because of the thermionic effect, thermionic emission and thermionic-field emission also contribute to charge injection. These injected charge carriers can be regarded to be caused by the ohmic conduction at the interface between metal and dielectrics [14], and these emissions are considered as a linear field-dependent function, as shown in Figure 4.

$$
J=\sigma E=\mu n q E
$$

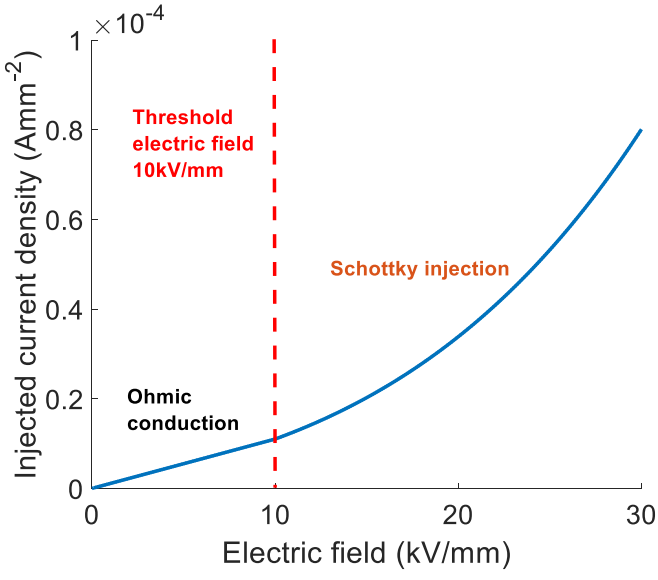

Figure 4. The simulated charge injection from anode as a function of electric field at $65^{\circ} \mathrm{C}$.

It is well known that temperature can have a great impact on charge injection at the interface and charge transport inside the dielectrics [15]. In previous model [9], the temperature gradient across the insulation is considered as being under the steady state, which is different from that of a practical HVDC cable operation. Here, a more realistic situation, i.e. the thermal transient of the cable, has been taken into account in order to simulate charge behaviors. Based on the time dependent heat transfer equation shown as following, the temperature distribution across the insulation at each time step could be obtained.

$$
\rho_{d} C_{p} \frac{\partial T}{\partial t}=\nabla \cdot(k \nabla T)+Q
$$

where $\rho_{d}$ is the material density; $C_{p}$ is the heat capacity at constant pressure; $k$ is the thermal conductivity; $Q$ is the heat generated from the conductor, of the form:

$$
Q=\frac{I^{2} \rho_{a l}}{A^{2}}
$$

where $A$ is the cross section area of the conductor; $I$ is the DC current; $\rho_{a l}$ is the resistivity of the cable core. The boundary condition is set to be convective heat flux at the outer surface of insulator.

\section{SIMULATION RESULTS}

Simulations have been performed on a medium voltage cable, the inner radius $\left(r_{i}\right)$ is $4.5 \mathrm{~mm}$, the outer radius $\left(r_{o}\right)$ is $9 \mathrm{~mm}$, giving an insulation thickness of $4.5 \mathrm{~mm}$. A positive voltage of $90 \mathrm{kV}$ is applied at the inner electrode and the average electric field inside the dielectric is about $20 \mathrm{kV} / \mathrm{mm}$. The parameters

\begin{tabular}{|c|c|c|c|}
\hline \multicolumn{2}{|c|}{ Parameter } & Value & Unit \\
\hline \multirow{2}{*}{$\begin{array}{l}\text { Injection barrier } \\
\text { heights }\end{array}$} & $\mathrm{W}_{\mathrm{ei}}$ for electrons & 1.27 & $\mathrm{eV}$ \\
\hline & $W_{\text {hi }}$ for holes & 1.16 & $\mathrm{eV}$ \\
\hline \multirow{2}{*}{$\begin{array}{l}\text { Trapping } \\
\text { coefficients }\end{array}$} & $\mathrm{B}_{\mathrm{e}}$ for electrons & 0.1 & $\mathrm{~s}^{-1}$ \\
\hline & $B_{h}$ for holes & 0.2 & $\mathrm{~s}^{-1}$ \\
\hline \multirow{2}{*}{$\begin{array}{l}\text { Trap depths (for } \\
\text { mobility) }\end{array}$} & $\mathrm{W}_{\mu \mathrm{e}}$ for electrons & 0.71 & $\mathrm{eV}$ \\
\hline & $w_{\mu h}$ for holes & 0.65 & $\mathrm{eV}$ \\
\hline
\end{tabular}
used in the simulation are given in Table 1, and these are mostly the ones that have been optimized from measurements achieved on space charge for a plane parallel LDPE [6].

Table 1. Parameters used for the simulations 


\begin{tabular}{|c|c|c|c|}
\hline Trap densities & $\begin{array}{l}\mathbf{n}_{\text {oet }} \text { for electrons } \\
\text { noht for holes }^{\text {ohe }}\end{array}$ & $\begin{array}{l}100 \\
100\end{array}$ & $\begin{array}{l}\mathrm{C} / \mathrm{m}^{3} \\
\mathrm{C} / \mathrm{m}^{3}\end{array}$ \\
\hline $\begin{array}{l}\text { Detrapping } \\
\text { barrier heights }\end{array}$ & $\begin{array}{l}\mathbf{W}_{\text {tre }} \text { for electrons } \\
\mathbf{W}_{\text {trh }} \text { for holes }\end{array}$ & $\begin{array}{l}0.96 \\
0.99\end{array}$ & $\begin{array}{l}\mathrm{eV} \\
\mathrm{eV}\end{array}$ \\
\hline
\end{tabular}

\subsection{COMPARISONS WITH THE PREVIOUS MODEL}

The first objective is to compare the simulated results with the ones of the previous model. In the previous work, the boundary condition is simply defined by a modified Schottky law at each electrode. However, the value of the injected current density may be different from the ones given by the Schottky injection law under a relatively high electric field. Additionally, when the applied electric field is lower than the critical value, this injected current density can be also different from the practical situation. In this modified model, the Schottky injection law dominates when the applied electric field exceeds the threshold, and the ohmic conduction takes over under the low field. A steady temperature gradient has been applied into the modelling, and the temperature distribution can be described using the following equation that derived from the thermal Ohm's Law, where $T\left(r_{i}\right)\left(65^{\circ} \mathrm{C}\right)$ and $T\left(r_{o}\right)\left(45^{\circ} \mathrm{C}\right)$ are the temperature at the inner and outer electrode respectively.

$$
T(r)=T\left(r_{o}\right)+\frac{\ln \left(\frac{r_{o}}{r}\right)\left(T\left(r_{i}\right)-T\left(r_{o}\right)\right)}{\ln \left(\frac{r_{o}}{r_{i}}\right)}
$$

After the application of voltage, both positive and negative charge carriers are observed next to the electrodes, with holes moving from the anode to the cathode and electrons moving oppositely, as shown in Figure 5. Compared with electrons that remain at the vicinity of the cathode, holes seem to penetrate into the volume much deeper. After $10000 \mathrm{~s}$, holes and electrons have already charged the bulk adequately, and holes' penetration is much deeper. The presence of homocharges strongly decrease the electric field at the inner electrode, leading to an approximately non-injecting electrode shortly after the application of voltage, as presented in Figure 6.

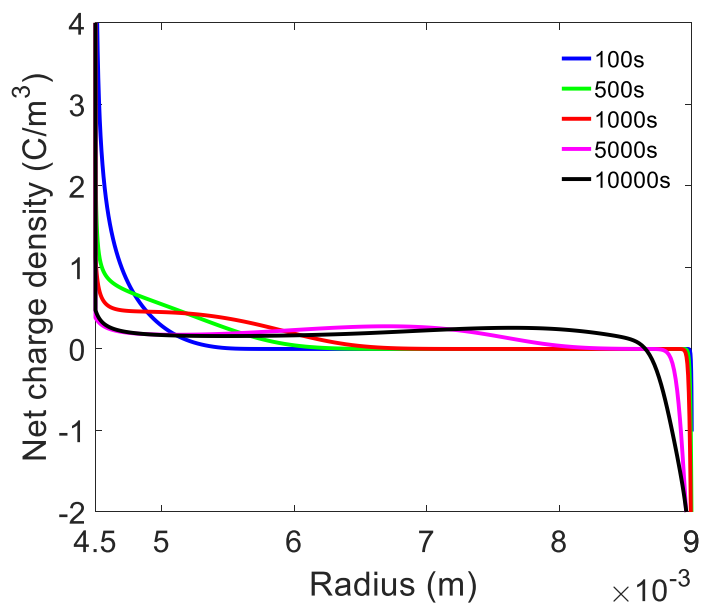

Figure 5. The simulated space charge density profiles in cylindrical geometry with a steady temperature gradient across the insulation. Anode to the left, cathode to the right.

With the time increasing, it can be observed that the maximal electric field location shifts from the anode to the cathode gradually. The reversing position of the maximal electric field, named stress inversion phenomenon, has already been observed experimentally by space charge measurements on MV cables [16]. The simulated results from the modified model, including the movement and distribution of charge carriers, the shift of the maximal electric field location and the value of the maximal electric field at $10000 \mathrm{~s}$ (reaching $33 \mathrm{kV} / \mathrm{mm}$ ), are still comparable with that from the previous ones, even though we modified the boundary condition at the electrodes [9][17]. This can be explained by the unchanged mobility (under the same temperature gradient) for each type of charge carriers and the similar amounts of injected holes and electrons as shown in Figure 7.

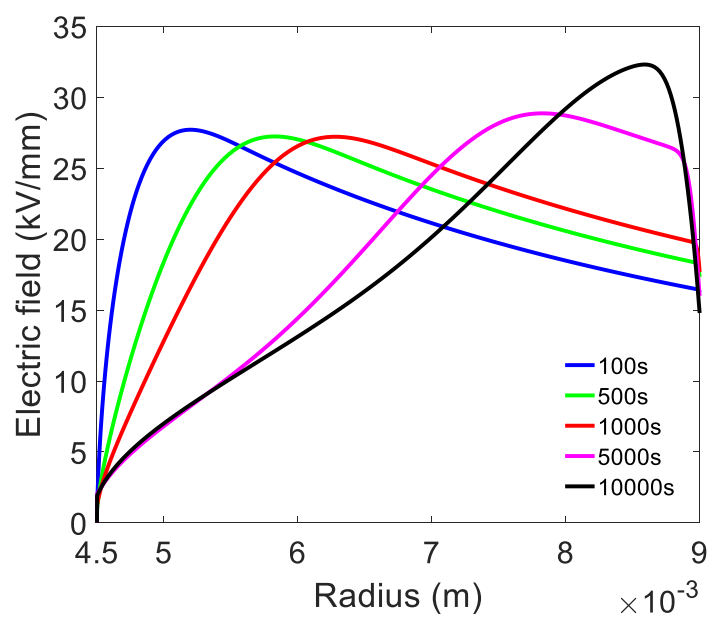

Figure 6. The electric field distributions in cylindrical geometry with a steady temperature gradient across the insulation. Anode to the left, cathode to the right.

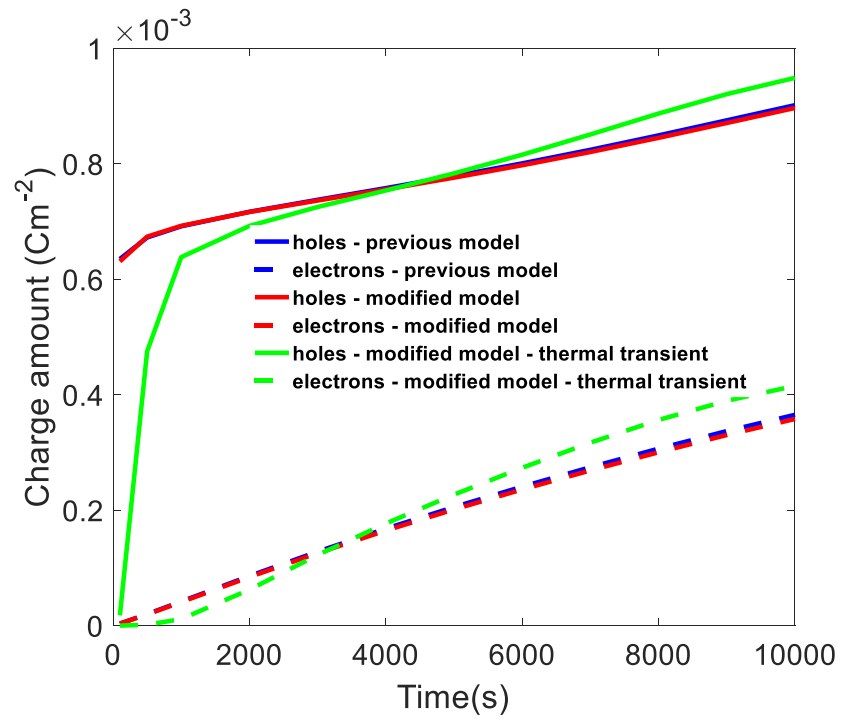

Figure 7. The amount of positive and negative charge carriers varying with time from different conditions (from $\mathrm{t}=100 \mathrm{~s}$ to $\mathrm{t}=10000 \mathrm{~s}$ ).

With the same temperature gradient, the amount of holes and electrons inside the bulk from the modified model and that from the previous are almost the same. Because of the high temperature $\left(65^{\circ} \mathrm{C}\right)$ at the inner electrode, most of the positive charge carriers are injected from the anode at the beginning, after that, only less positive charge carriers are injected as the electric field at the anode has been decreased due to the injected homocharges. The situation is different in the case of thermal transient states as demonstrated in the next section. 


\subsection{THERMAL TRANSIENT}

In order to investigate space charge behaviour and field distribution in HVDC cable insulation under more realistic conditions, a transient temperature gradient is introduced into the model. When a DC current of $350 \mathrm{~A}$ is applied to the core of cable, a quasi-equilibrium can be reached after 2 hours with a temperature gradient of $\sim 20^{\circ} \mathrm{C}$, as shown in Figure 8. Here, we aim to analyse the thermal transient effects on space charge behaviours and make comparisons with the steady state.

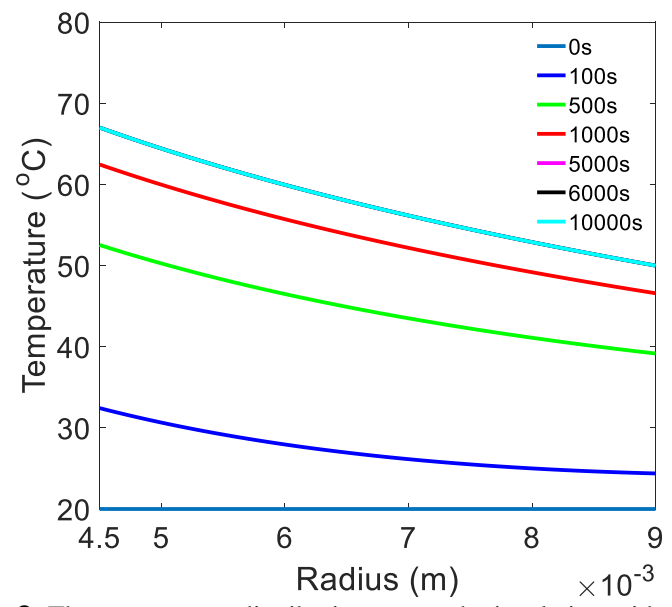

Figure 8. The temperature distribution across the insulation with a current of 350 A flowing in the core of cable.

Figure 9 presents the space charge distribution within the insulation evolving with time. Compared with the results obtained under the steady temperature gradient, big differences can be found in the first $1000 \mathrm{~s}$. The electrons remain near the cathode, but the holes do not seem to penetrate into the volume until $t=1000 \mathrm{~s}$, and the movement of charge carriers at $\mathrm{t}=1000 \mathrm{~s}$ is similar with the $100 \mathrm{~s}$ result shown in Figure 5. This can be explained by the huge thermal impacts on charge injection and the mobility of charge carriers. However, after $5000 \mathrm{~s}$, the space charge distribution seems alike with the result shown in Figure 5 and almost the same at the end of $10000 \mathrm{~s}$.

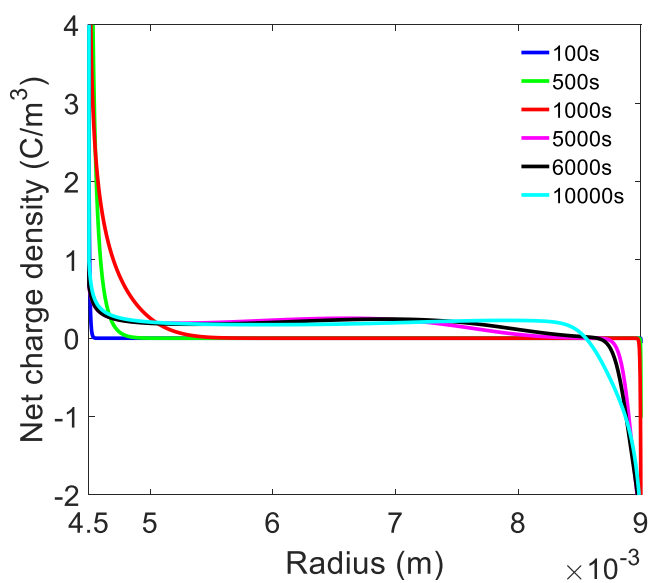

Figure 9. The simulated space charge density profiles as a function of radius for different polarization times. Applied voltage $90 \mathrm{kV}$, with a current of 350 A flowing in the conductor.

The corresponding electric field distribution are shown in Figure 10. It can be observed that the decrease of the electric field at the anode in the beginning is not as obvious as that shown in Figure 6, and the electric field at the anode drops to nearly zero until $\mathrm{t}=1000 \mathrm{~s}$. The shift of the maximal electric field location in the first $1000 \mathrm{~s}$ is not as quick as that shown in Figure 6. However, at the end of the polarization, the electric field distribution is almost same. There is only a little difference on the value (about $1 \mathrm{kV} / \mathrm{mm}$ ) and the location (about $0.2 \mathrm{~mm}$ ) of the maximal electric field at $\mathrm{t}=10000 \mathrm{~s}$, and differences on the total amount of injected charge carriers are regarded as the main reason.

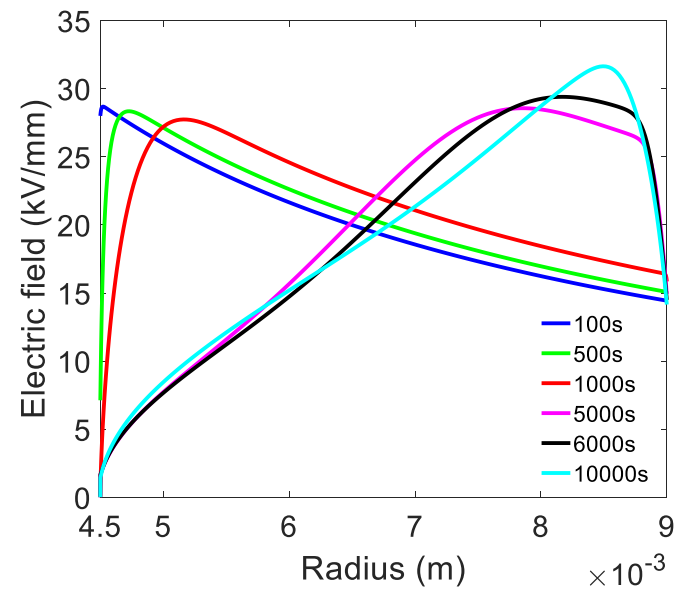

Figure 10. The electric field distribution for different polarization times. Applied voltage $90 \mathrm{kV}$, with a current of 350 A flowing in the conductor. Anode to the left, cathode to the right.

\subsection{THERMAL TRANSIENT WITH A CHANGING CURRENT}

In order to further study the impact of thermal transient effects, a changing current is applied into the cable conductor. When a DC current of $250 \mathrm{~A}$ is applied into the core of cable, a stationary state of $\sim 10{ }^{\circ} \mathrm{C}$ temperature gradient $\left(45-35^{\circ} \mathrm{C}\right)$ across the insulation can be reached after 2 hours. Firstly, the current flow in the conductor is set to be $250 \mathrm{~A}$ in the first 5000 $\mathrm{s}$, then change into $350 \mathrm{~A}$ for the rest $5000 \mathrm{~s}$. In this situation, the temperature gradient inside the bulk is raised and maintained in the first period, and then raised and maintained again in the rest of time, as described in Figure 11.

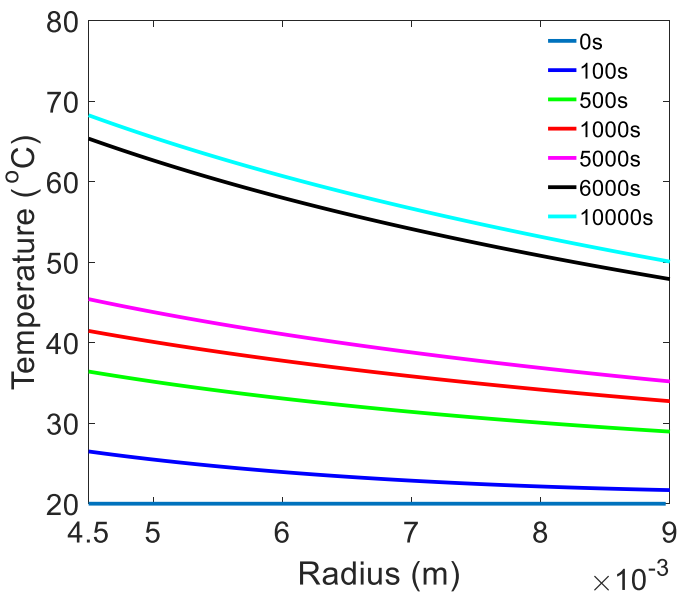

Figure 11. The temperature distribution across the insulation with a changing current (250 A-350 A) flowing in the core of cable.

The space charge density profiles as a function of radius calculated by this modified model under this transient temperature distribution are shown in Figure 12. Both the holes 
and electrons can be seen near the cathode at the beginning, however, in the first $1000 \mathrm{~s}$, both of positive and negative charge carriers remain close to the electrodes and do not penetrate into the volume. Until $\mathrm{t}=5000 \mathrm{~s}$, holes' penetration can be observed but it is far less deep than the results shown in Figure 9. After $5000 \mathrm{~s}$, the temperature across the insulation is increased significantly, so is the mobility for each type of charge carriers, therefore both holes and electrons can move into the bulk quickly. Compared with the results shown in Figure 9, the space charge distribution at the end is almost the same. This is particularly surprising as the temperature distribution is very different from which shown in Figure 8 in the first $5000 \mathrm{~s}$, as if the stationary state of temperature distribution can determine the final charge distribution across the insulation.

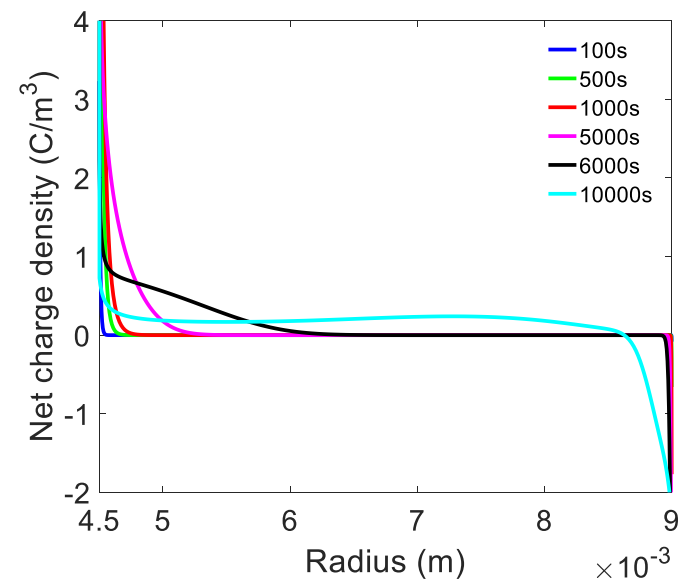

Figure 12. The simulated space charge density profiles as a function of radius for different polarization times, with a changing current (250 A-350 A) flowing in the conductor

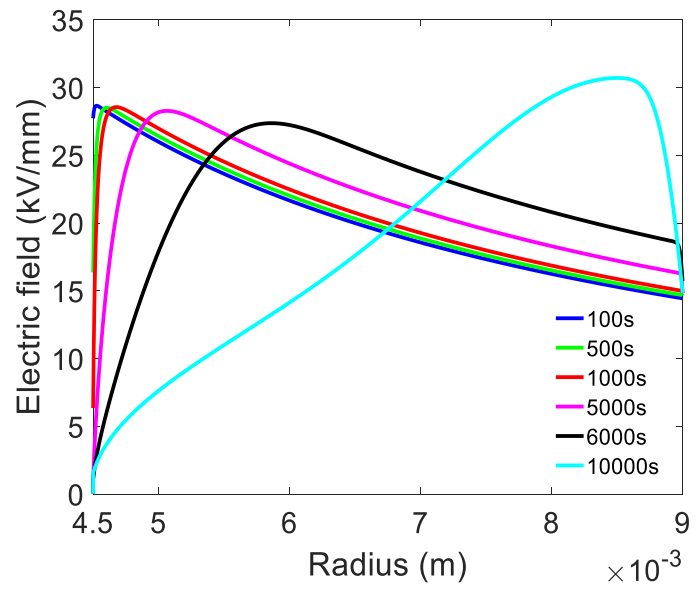

Figure 13. The electric field distribution for different polarization times, with a changing current (250 A-350 A) flowing in the conductor. Anode to the left, cathode to the right.

Some differences still can be found in the electric field distribution, as presented in Figure 13. We can observe that the maximal electric field location remains at the inner electrode in the first period $(t<5000 \mathrm{~s})$. However, when the general temperature increases and the temperature gradient reaches $20{ }^{\circ} \mathrm{C}$, the maximal electric field location shifts to the outer electrode gradually. In other words, the stress inversion phenomenon cannot happen in relatively low temperature and small temperature gradient condition. Only when it comes to the higher temperature and the larger temperature gradient, the stress inversion can take place. Additionally, the value of the maximal electric field at the end is smaller than that shown in Figure 10, and this could be explained by the difference on the amount of injected charge carriers inside the insulation due to the different thermal condition.

In another case, the current flow is set to be $350 \mathrm{~A}$ in the first $5000 \mathrm{~s}$ and then change into $250 \mathrm{~A}$ in the rest of time. Therefore, the general temperature inside the volume is raised and maintained in the first $5000 \mathrm{~s}$, and then it is descended and maintained in the rest of time, as shown in Figure 14.

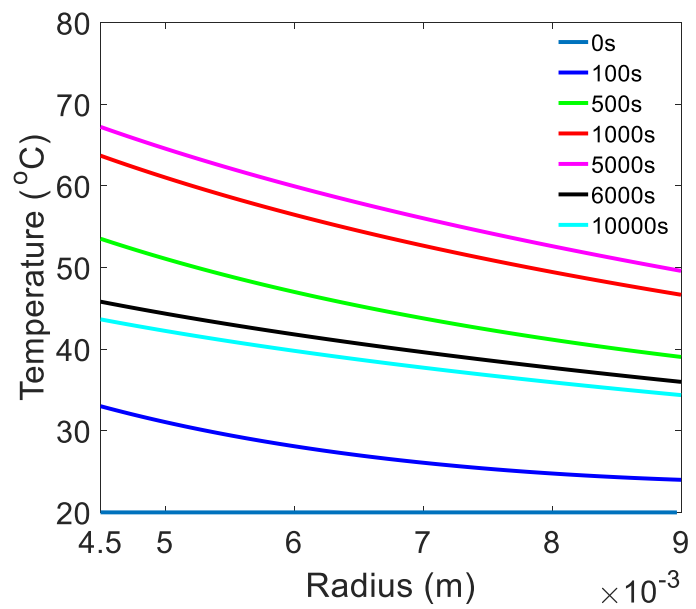

Figure 14. The temperature distribution across the insulation with a changing current (350 A-250 A) flowing in the core of cable.

Figure 15 shows the space charge density profiles calculated by this modified model with a changing current flowing in the core of cable. In the first period, both positive and negative charge carriers can penetrate into the volume, similarly with the result in Figure 9. Interestingly, after the current flowing in the conductor is reduced to $250 \mathrm{~A}$, the distribution and the amount of charge carriers almost remain unchanged, and it seems to be frozen at the same position. The electric field distributions also behave in keeping with the space charge density profiles, as presented in Figure 16.

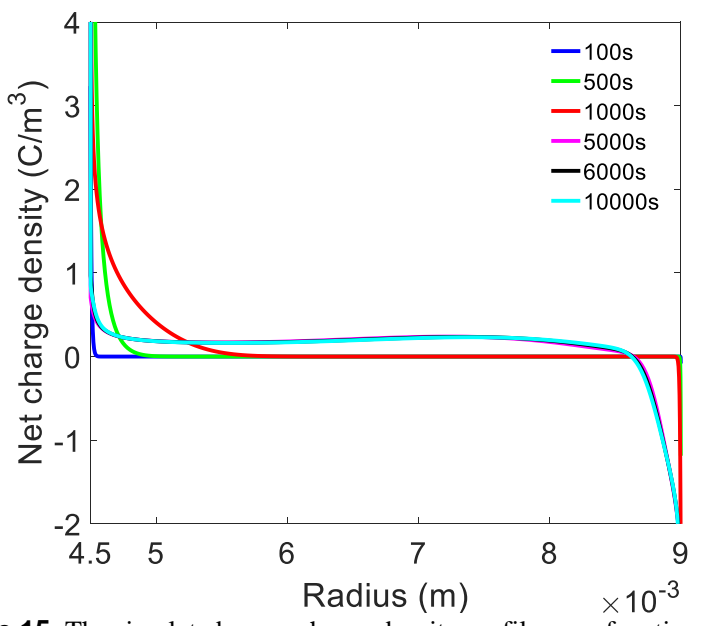

Figure 15. The simulated space charge density profiles as a function of radius for different polarization times, with a changing current (350 A-250 A) flowing in the conductor. 
The maximal electric field location shifts from the inner electrode to the outer electrode in the first $5000 \mathrm{~s}$, and then the location and the value of the maximal electric field remain consistent in the rest of time. It may be explained by the following reasons. Firstly, the mobility of both kinds of charge carriers drops significantly with the decreasing of temperature. The movement of charge carriers become extremely slow under this relatively low temperature condition. Secondly, there are very few charge carriers injected from the electrodes due to the low electric field at the anode and the low temperature at the cathode after $\mathrm{t}=5000 \mathrm{~s}$.

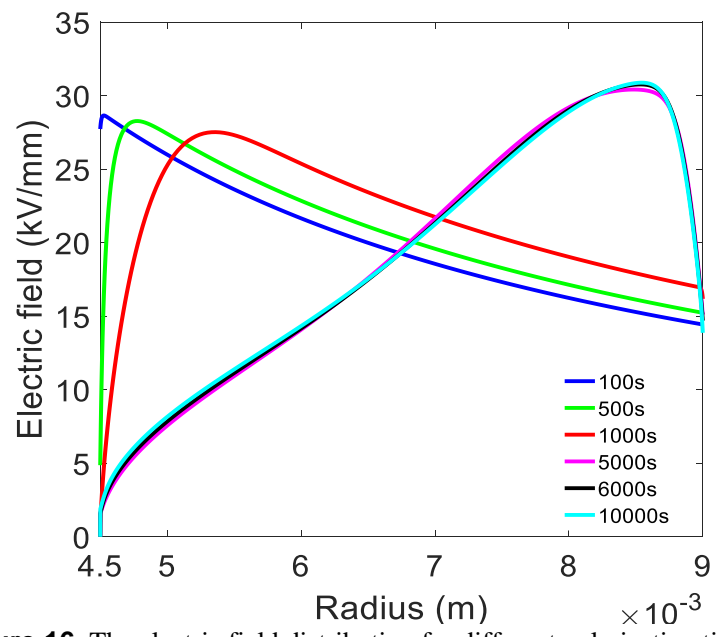

Figure 16. The electric field distribution for different polarization times, with a changing current (350 A-250 A) flowing in the conductor. Anode to the left, cathode to the right.

It is worth noting that in this modified model, the maximal electric field location does not change with the decreasing temperature across the insulation, in other words, the stress inversion phenomenon still presents even with the temperature gradient dropping inside the bulk. This simulation result still need to be further proven by the space charge measurement experiments, which are being prepared in Tony Davis High Voltage Laboratory and comparisons on anticipating field distribution also should be made between this model and the models which based on macroscopic parameter conductivity.

\section{CONCLUSION}

A modified bipolar charge transport model has been used to simulate space charge behavior and electric field distribution in a cable insulation in the presence of temperature gradient. A threshold electric field has been introduced into the model to redefine the charge injection from the electrodes. The following conclusions can be drawn.

The well-known stress inversion in HVDC cable in the presence of temperature gradient has been observed.

A transient temperature distribution calculated by heat diffusion equation has been applied across the insulation in this model, in order to obtain the space charge profiles in practical HVDC applications. The thermal transient shows its impacts on the amount of injected charge carriers, and it can slow down the movement of charge carriers and the electric field variation. Although after reaching the stationary state, the results of thermal transient are similar with that of a steady temperature gradient, they still present some differences on the value and the location of the maximal electric field at the end of polarization. Additionally, with this modified model, we found that the stress inversion phenomenon only take place with a relatively higher temperature and a larger temperature gradient $\left(>20^{\circ} \mathrm{C}\right)$. Moreover, the maximal electric field location did not change when we decreased the current flowing in the conductor from $350 \mathrm{~A}$ to $250 \mathrm{~A}$. This result should be further studied and compared with that of the other models which are based on the macroscopic conductivity data as a function of electric field and temperature.

\section{REFERENCES}

[1] G. Chen, M. Hao, Z. Xu, A. Vaughan, Cao, Junzheng, and H. Wang, "Review of High Voltage Direct Current Cables," 10 Csee J. Power Energy Syst., vol. 1, no. 2, pp. 9-21, 2015.

[2] H. Ghorbani, M. Jeroense, C.-O. Olsson, and M. Saltzer, "HVDC cable systems - highlighting extruded technology," IEEE Trans. Power Deliv., vol. 29, no. 1, pp. 414-421, 2014.

[3] T. L. Hanley, R. P. Burford, R. J. Fleming, and K. W. Barber, "A general review of polymeric insulation for use in HVDC cables," IEEE Electr. Insul. Mag., vol. 19, no. 1, pp. 13-24, 2003.

[4] M. S. Khalil, "International research and development trends and problems of HVDC cables with polymeric insulation," IEEE Electr. Insul. Mag., vol. 13, no. 6, pp. 35-47, 1997.

[5] P. H. F. Morshuis, R. Bodega, D. Fabiani, G. C. Montanari, L. A. Dissado, and J. J. Smit, "Calculation and Measurement of Space Charge in MV-size Extruded Cables Systems under Load Conditions," in Solid Dielectrics, 2007. ICSD'07. IEEE International Conference on, 2007, pp. 502-505.

[6] S. Le Roy, G. Teyssedre, C. Laurent, G. C. Montanari, and F. Palmieri, "Description of charge transport in polyethylene using a fluid model with a constant mobility: fitting model and experiments," J. Phys. D. Appl. Phys., vol. 39, no. 7, p. 1427, 2006.

[7] G. Chen and S. H. Loi, "Space charge modelling in solid dielectrics under high electric field based on double charge injection model," MRS Online Proc. Libr. Arch., vol. 889, 2005.

[8] S. Le Roy, P. Segur, G. Teyssedre, and C. Laurent, "Description of bipolar charge transport in polyethylene using a fluid model with a constant mobility: model prediction," J. Phys. D. Appl. Phys., vol. 37, no. 2, pp. 298-305, 2004.

[9] S. Le Roy, G. Teyssedre, and C. Laurent, "Modelling space charge in a cable geometry," IEEE Trans. Dielectr. Electr. Insul., vol. 23, no. 4, pp. 2361-2367, 2016.

[10] M. Fu, G. Chen, and A. E. Davies, "Space Charge Measurements in Cables Using the Pea," pp. 219-222, 2001.

[11] G. Chen and Z. Xu, "Charge trapping and detrapping in polymeric materials," J. Appl. Phys., vol. 106, no. 12, pp. 1-5, 2009.

[12] K. C. Kao, Dielectric phenomena in solids. Academic press, 2004

[13] N. Liu, C. Zhou, G. Chen, and L. Zhong, "Determination of threshold electric field for charge injection in polymeric materials," Appl. Phys. Lett., vol. 106, no. 19, p. 192901, 2015.

[14] G. C. Montanari, G. Mazzanti, F. Palmieri, A. Motori, G. Perego, and S. Serra, "Space-charge trapping and conduction in LDPE, HDPE and XLPE," J. Phys. D. Appl. Phys., vol. 34, no. 18, p. 2902, 2001.

[15] G. Teyssedre and C. Laurent, "Charge transport modeling in insulating polymers: From molecular to macroscopic scale," IEEE Trans. Dielectr. Electr. Insul., vol. 12, no. 5, pp. 857-874, 2005.

[16] T. T. N. Vu, G. Teyssedre, B. Vissouvanadin, S. Le Roy, C. Laurent, M. Mammeri, I. Denizet, "Field distribution in polymeric MV-HVDC model cable under temperature gradient: simulation and space charge measurements," Eur. J. Electr. Engg., vol. 17, pp. 307-325, 2014.

[17] D. Fabiani G. Montanari, C. Laurent, G. Teyssedre, P. H. F. Morshuis, R. Bodega, L. A. Dissado, "HVDC cable design and space charge accumulation. part 3: effect of temperature gradient," IEEE Electr. Insul. Mag., vol. 24, no. 2, pp. 5-14, 2008. 


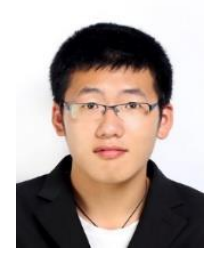

Yunpeng Zhan was born in in China in 1994. He received his B.Eng. degree (2016) in electronic science from Xiamen University, China. Since 2016, he has been a Ph.D student in University of Southampton, UK. His main research interests are space charge, insulation materials and HVDC cables.

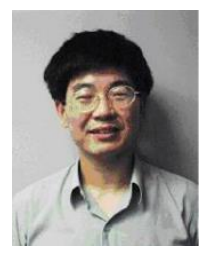

George Chen (SM'11) was born in China in 1961. He received his B.Eng (1983) and M.Sc (1986) degrees in electrical engineering from Xi'an Jiaotong University, China. After he obtained the Ph.D. degree (1990) from the University of Strathclyde, UK, on the permanent changes in the electrical properties of irradiated low-density polyethylene, he joined the University of Southampton as a postdoctoral research fellow and subsequently became a senior research fellow. In 1997 he was appointed as a research lecturer and promoted to a Reader in 2002. He is now a professor of high voltage engineering at the University of Southampton and a visiting professor of Xi'an Jiaotong University. He has developed a wide range of interests in high voltage engineering and electrical properties of materials and published over 300 papers. $\mathrm{He}$ is active in HVDC systems and involved with technical working groups in both IEEE and CIGRE.

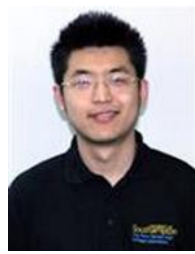

Miao Hao was born in China in 1987. He received his B.Eng. degree (2009) from Xi'an Jiaotong University, China. After he obtained his M.Sc. degree (2011) and Ph.D. degree (2015) from University of Southampton. He is now a postdoctoral research fellow in the University of Southampton. His main research interests include space charge and ageing mechanism in dielectrics for HVDC applications. 